\title{
Un dizionario di citazioni
}

A Dictionary of Quotations

Un dictionnaire de citations

\section{Alberto Cadioli}

\section{OpenEdition}

\section{Journals}

\section{Edizione digitale}

URL: https://journals.openedition.org/cei/6596

DOI: 10.4000/cei.6596

ISSN: 2260-779X

\section{Editore}

UGA Éditions/Université Grenoble Alpes

\section{Edizione cartacea}

ISBN: 978-2-37747-173-7

ISSN: $1770-9571$

\section{Notizia bibliografica digitale}

Alberto Cadioli, «Un dizionario di citazioni», Cahiers d'études italiennes [Online], 30 | 2020, online dal 01 mars 2020, consultato il 21 septembre 2021. URL: http://journals.openedition.org/cei/6596 ; DOI: https://doi.org/10.4000/cei.6596

Questo documento è stato generato automaticamente il 21 septembre 2021.

(c) ELLUG 


\title{
Un dizionario di citazioni
}

\author{
A Dictionary of Quotations \\ Un dictionnaire de citations
}

Alberto Cadioli

1 C'è una curiosa 'vicinanza' tra due citazioni 'lontanissime' tra loro: l'incipit del racconto di Gesualdo Bufalino L'ingegnere di Babele ${ }^{1}$ e un profilo di Walter Benjamin tracciato da Hanna Arendt.

2 Scrive Bufalino, introducendo la figura del protagonista del suo racconto, impegnato nell'ambizioso progetto di allestire un grande «Dizionario di citazioni»:

Lo vedevo ogni giorno al bar per il caffè delle nove, e ve lo ritrovavo la sera, seduto allo stesso tavolo di marmo, con una sporta di libri accanto e una biro in allarme fra l'indice e il medio. [...] Febbrile lettore, s'interrompeva di quando in quando per ricopiare brevemente una frase su un notes, indi ricominciava. Un cliente senza nome, mi dissero, ma al bar lo chiamavano Robinson ${ }^{2}$.

3 Hanna Arendt aveva a sua volta sottolineato come «negli anni Trenta niente era più caratteristico per lui [Walter Benjamin] dei piccoli quadernetti di appunti rilegati in nero che portava sempre con sé e in cui trascriveva instancabilmente in citazioni ciò che la vita o il leggere quotidiano gli offriva» ${ }^{3}$. E anche Theodor Adorno, in una sua osservazione molto nota, ricordava che Benjamin avrebbe voluto consegnare la propria opera più importante a un libro composto solo di citazioni ${ }^{4}$.

4 La coincidenza si ferma qui, e non si hanno testimonianze che Bufalino conoscesse l'opera di Benjamin o le pagine che descrivono il suo continuo ricorso alle parole degli altri. E tuttavia l'accostamento del progetto di Robinson (confinato nel mondo della finzione) e di quello di Benjamin (rimasto un ideale) permette di gettare uno sguardo più approfondito sull'idea bufaliniana di un libro di citazioni, e vedere da un punto di vista forse poco utilizzato il rapporto della scrittura di Bufalino con i testi che, lettore onnivoro $^{5}$, aveva avuto in mano, un rapporto sul quale la critica ha insistito fin dalla pubblicazione del libro di esordio, Diceria dell'untore.

5 Gli studiosi di Benjamin che si sono occupati dello scrittore tedesco in riferimento al suo uso della citazione ${ }^{6}$ hanno sottolineato più volte come l'inserimento in un proprio 
testo di un frammento di parole altrui - anche quando si trattava di un saggio monografico su uno scrittore e dunque era inevitabile riportarne alcune frasi (è il caso del famoso saggio su $\mathrm{Kafka}^{7}$ ) - non sia da considerare un recupero storicizzante, ma il riutilizzo, con atteggiamento scarsamente filologico, di materiali già esistenti. Da qui l'indifferenza anche per la tipologia del frammento: tratto da qualunque pagina, sia essa di qualità o di consumo, di scrittore noto o di autore oscuro, ciò che importava a Benjamin era la funzione assunta dalla citazione secondo la personale necessità dello scrittore, in dialogo con i lettori suoi contemporanei.

Disinteressato allo storicismo e ai suoi metodi, Benjamin intende recuperare una dimensione appartenente a un tempo considerato non raggiungibile (scrive Gabriele Scaramuzza: «La citazione è intrisa del senso dell'irrecuperabile e dell'appuntamento mancato, più che dell'incontro riuscito» $\left.{ }^{8}\right)$, perché i frammenti isolati dal testo d'origine sono funzionali alla costruzione di nuovi testi: «la citazione è trouvaille e materiale da costruzione al tempo stesso»' ${ }^{9}$. Da qui una pratica che trascura la fedeltà e la correttezza e può arrivare alla manipolazione, da qui l'attribuzione ad alcuni di frasi di altri, fino alla vera e propria falsificazione, come gli studiosi benjaminiani hanno sottolineato. Lo scritto su Kafka è emblematico: «Se il saggio si costruisce per interventi destrutturanti che spiazzano il lettore, questo si fa particolarmente evidente nell'uso delle citazioni; ma la loro decontestualizzazione anche riscopre in esse sensi letterali perduti, ne rivela strati rimossi, dà loro nuove voci, colori prima assenti - e forse propositivi» $»^{10}$.

7 Il grande progetto di Robinson del racconto di Bufalino - affidato a un «album infolio», del tipo di quelli nei quali «si conservano i progetti degli ingegneri» ${ }^{11}$, ragion per cui il suo ideatore può essere definito borgesianamente «l'ingegnere di Babele»sembra andare in direzione esattamente opposta: l'allestimento del grande «Dizionario di citazioni», che potrebbe avere il titolo di Dispar et unum, o La Mela d'Eva, o L'abbecedario supremo, ma che, in quanto «centone» di testi «ritagliati» (e forse Robinson, a suo tempo bibliotecario, era stato licenziato «per un oscuro affare di volumi, chi diceva rubati, chi mutilati con le forbici» ${ }^{12}$ ) ha una funzione precisa: «Non c'è altro vademecum o scatola nera a disposizione, chi voglia mettere in salvo per i posteri della post-storia una reliquia almeno di quel che l'uomo ha saputo pensare confusamente nei secoli ${ }^{13}$.

8 La citazione, dunque, come «sineddoche», la "particola in cambio del tutto» ${ }^{14}$; e l'idea di un libro di citazioni nel quale «ridurre in un sol tomo di mille pagine la quintessenza e il mistero, la verità e la bellezza d'ogni scrittura: Libro dei Libri, solitario alambicco che converte in oro massiccio le infinite cascate d'inchiostro scorse nel mondo da quando qualcuno scrisse sulla sabbia con un dito la prima parola di spavento o d'amore.... ${ }^{15}$.

9 Le citazioni, dunque, a questa altezza del racconto, non si pongono come commento al presente o come esercizio di critica nei confronti di un testo illuminato di luce nuova che, grazie all'accostamento delle citazioni, favorisce sguardi inediti; quanto piuttosto sono la summa «ritagliata» che racchiude l'intero sapere, del quale è necessario dare testimonianza anche per lacerti, non potendolo custodire nella sua complessità. L'ansia della sovrabbondanza di libri da leggere, la consapevolezza dell'impossibilità di arrivare a conoscere tutto («anche morendo assai tardi, moriremo troppo presto, con entrambi i pugni pieni di mosche.... $\left.{ }^{16}\right)$, spinge Robinson a tramandare i frammenti di una civiltà ingigantita, imitando quegli antichi che avevano «il costume pio [...] di chiudere accanto al sepolto, per viatico funerario e riassunto della sua vita, granelli di frumento, pettini d'osso e monili.... ${ }^{17}$. 

avrebbe potuto concludersi. In realtà la narrazione si riapre subito: «Robinson impazzì cinque settimane dopo. Di colpo, come uno lo fulmina un embolo» ${ }^{18}$. La conseguenza è subito visibile all'io narrante, quando gli vengono presentati «gli spogli del giorno prima»: «Vidi subito che si trattava di apocrifi, citazioni da opere inesistenti, costruite con un gusto così smaccato dell'irrisione da doversene escludere ogni uso che non fosse salottiero o goliardico» ${ }^{19}$.

11 La follia si rivela tuttavia nella serietà di Robinson, che non «ammetteva che si trattasse di falsi, bensì sosteneva che erano scoperte sue, da opere ghiotte, introvabili, ignote alle bibliografie $»^{20}$. Le citazioni da Lieti tropici di Venerdì (con evidente rimando a Gulliver e a Lévi-Strauss) o da Storia dell'automobile di Tristan Tzara e Isotta Fraschini, o ancora dalla Chanson de Roland Barthes, disorientano l'io narrante. quella pratica dell'intertestualità che la critica ha ampiamente riconosciuto nelle pagine di Bufalino, sottolineando, per ricorrere solo alle parole di Maria Corti, il «virtuosismo» e la «molteplicità dei piani di scrittura» ${ }^{21}$, l'uno e l'altra contigui al contesto barocco dentro il quale possono entrare, «nel sublime gioco dello stile e della citazione occulta» ${ }^{22}$, tutti i testi che lo scrittore ha compulsato nelle sue letture.

L'accumulo di citazioni (tra le quali anche citazioni false, scritte 'à la manière de'), è richiamato ormai come componente fondamentale della letteratura post-moderna: qui interessa il fatto che sia centrale anche nella scrittura di Bufalino, e a questo proposito, più che le molte pagine critiche dedicate allo scrittore, può essere utile ricordare che le carte autografe riportano spesso, sullo stesso foglio dattiloscritto, liste nomi di scrittori, le cui opere sono sia punto di riferimento sia serbatoio di citazioni da inserire o da «riscrivere» nel testo in elaborazione ${ }^{23}$.

14 La follia di Robinson permette però di inoltrarsi in territori diversi rispetto a quello dell'intertestualità (e delle 'fonti', secondo una più antica dizione), e di mettere a fuoco non solo o non tanto il gioco del rimando ad altri testi, palesi o nascosti, quanto piuttosto di precisare una delle idee espresse da Bufalino riguardo alle citazioni, e approfondire uno dei compiti loro affidato ${ }^{24}$.

15 Lo scartafaccio sporgente dalla tasca di Robinson con i nuovi spogli destinati alle sue cartellette si offriva ancora come «la sottile zattera di carta con cui [Robinson] s'apprestava una volta di più ad affrontare il diluvio» ${ }^{25}$, ma il progetto iniziale era, non consapevole il suo autore, ormai cambiato, soprattutto per la moltiplicazione esponenziale delle citazioni false. L'obiettivo perseguito, coscientemente o meno, non riguardava più la salvezza della storia da raggiungere grazie ad alcuni frammenti sopravvissuti dal passato, quanto la salvezza di sé stessi dal mondo circostante, percepito come sempre più distante.

16 Ed è a questo punto che, paradossalmente, la chiusura del racconto L'ingegnere di Babele si indirizza verso la posizione benjaminiana: la citazione diventa funzionale allo straniamento del lettore, e quindi portatrice di uno sguardo nuovo, per introdurre il quale poco importa se la trascrizione sia corretta o imprecisa, se le parole riportate siano di una vera citazione o scritte appositamente in forma di citazione. Benjamin costruiva le sue pagine di citazioni per ragioni conoscitive, per aumentare cioè il grado di conoscenza o di consapevolezza del lettore; il personaggio di Bufalino, quando è 


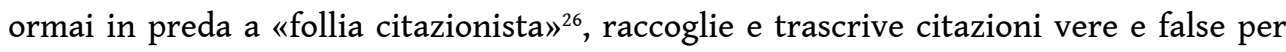
ragioni di salvezza individuale.

E tuttavia l'idea originaria dell'«ingegnere di Babele» - quella di mettere in salvo il sapere del mondo - esce dalle pagine del racconto ed entra, con una nuova forma, nel Dizionario dei personaggi di romanzo, il libro che Bufalino dà alle stampe nel 1982. Il Dizionario, che porta il sottotitolo «Da don Chisciotte all'Innominabile» ${ }^{27}$, si apre con una dichiarazione che potrebbe essere tratta direttamente dal progetto di Robinson (cui lo lega quanto meno il rimando al termine «dizionario»), prima che la follia insorgesse. Scrive dunque Bufalino nell'«Introduzione», presentando la figura del «compilatore di antologie» (per indicare il quale si parla anche di «appassionato di squartamenti»: e si ricordi che Robinson era stato accusato di tagliare materialmente i volumi della biblioteca presso la quale lavorava):

Di lingua subdola, di mano spiccia, di smisurata superbia, egli meriterebbe il bando dalle pubbliche biblioteche, se la sua opera non si rivelasse provvidenziale nelle emergenze di apocalisse prossima ventura, quando non ci vuol meno dei suoi coltelli da cuciniere per fornire ai clienti delle catacombe il Libro dei Libri, surrogatorio d'ogni altro, tascabile lingotto di lacerti pressati, da nascondere in fretta nella valigia, fra una borraccia e il rasoio, subito dopo lo squillo della prima tromba del cherubino ${ }^{28}$.

Il lessico introdotto è strettamente vicino a quello di Robinson: là il Diluvio, qui l'apocalisse; là le forbici, qui l'immagine del cuciniere con i coltelli. L'obiettivo è lo stesso, un Libro dei Libri che, sotto forma di «lingotto di lacerti», salvi il sapere, anche se con scelte individuali, come viene precisato subito sotto: «[...] a questo punto prudenza vuole che ognuno si nomini da solo Deucalione e Noè, e metta mano a salvare almeno un compendio dello scibile che più gli preme» ${ }^{29}$. Proprio questo lessico condiviso tra il racconto e le pagine saggistiche dell'introduzione, proprio la manifestazione di un territorio comune tra il racconto e il nuovo libro, permette di leggere il Dizionario dei personaggi di romanzo come una grande raccolta di citazioni, soprattutto se ciò che permette di parlare di «citazione» non è la lunghezza del testo riportato, ma la sua estrazione dal contesto di provenienza, da un lato, il suo inserimento in un nuovo contesto, dall'altro. I testi raccolti nel Dizionario dei personaggi di romanzo hanno misure diverse (alcuni sono lunghi), ma ciascuno di essi è il «ritaglio» che isola un singolo passo dall'opera cui appartiene. Bufalino seleziona e rende autonome quelle frasi che permettono di dar conto dei personaggi più memorabili della letteratura moderna, e assegna loro una funzione di conservazione, la stessa che assumono «un fossile» $\mathrm{o}$ «un calco pompeiano»" ${ }^{30}$, frammenti che dentro di sé portano il mondo dal quale provengono.

Inevitabilmente (e lo dichiara lo stesso Bufalino), la scelta dei personaggi è arbitraria, essendo impossibile un'assoluta neutralità: ma l'autore - come lui stesso sottolinea in un'«Avvertenza» - «per come ha potuto, l'ha cercata», e ha fondato le proprie scelte («il suo canone») obbedendo «meno alle ragioni del gusto privato che a quelle del comune consenso» ${ }^{31}$.

Dalla bizzarria di Robinson si passa dunque alla lucidità di Bufalino, che per ogni personaggio di romanzo (collocato in un arco cronologico che va dal 1605, l'anno di Don Chisciotte e Sancio Panza, al 1953, l'anno dell'Innominabile di Beckett), trascrive un passo e lo introduce con poche righe di commento, che a loro volta rimandano ad altre letture, ad altre citazioni esplicite o implicite. Emblematica la conclusione del primo 
commento, nel quale Don Chisciotte viene definito l'unico «per cui si possa dire, contro Goya, che il sonno della ragione genera angeli ${ }^{32}$.

21 L'obiettivo - e si può dire il risultato finale - è la composizione, per usare ancora le parole di Bufalino, di «un album di sinopie burocratiche e utilitarie; qualcosa come un casellario giudiziario o una vetrina di farfalle, ciascuna col suo spillino nell'addome imbalsamato; se non uno di quei campionari di veneri venali che si esibiscono in visione ai commendatori in trasferta» ${ }^{33}$.

Come per ogni libro, tuttavia, è la lettura a generare il senso:

Toccherà infine al lettore, se Dio vuole, giustificare manomissioni così disinvolte, quando sappia giovarsene a ricomporre, non diversamente che da un fossile o da un calco pompeiano, le fattezze della figura originaria; e a cucirsi con le singole pezze un solo grande romanzo-arlecchino, un film-monstre dall'ineguagliabile cast ${ }^{34}$.

Il compito del lettore del Libro dei Libri, a questo punto, tuttavia, va oltre quello previsto dall'«ingegnere di Babele» e oltre quello indicato da Bufalino nell'introduzione al Dizionario, quando segue l'evoluzione del personaggio romanzesco tracciandone la storia attraverso «un monotono esercito di spettri»"

L'insieme delle citazioni del dizionario viene infatti indicato ora come possibile generatore di altre modalità di lettura. Le pagine del Dizionario possono essere infatti utilizzate dal lettore come materiale per un nuovo romanzo che viene via via componendosi nella sua testa nel corso della lettura, e così le citazioni si incastrano una nell'altra per offrire un'esperienza nel presente del lettore. Il romanzo può sopravvivere, nelle catacombe dopo l'apocalissi, solo grazie a un «libro-arlecchino» fondato sui personaggi: su un libro di citazioni, dunque.

Dopo avere dato un'ennesima definizione del suo Dizionario - un «breviario», quasi a scandire le ore del giorno del lettore con una lettura-meditazione - Bufalino sottolinea come l'accostamento di citazioni possa dare origine non solo a un nuovo libro, ma ad un nuovo mondo. Si tratta di uun universo parallelo e sussidiario al nostro e probabilmente più felice, quali che siano i sospetti e le incredulità che lo insidiano» ${ }^{36}$ : un «territorio invisibile», che tuttavia costituisce «la nostra patria più vera»"

Con le ultime righe dell'introduzione al Dizionario l'obiettivo della citazione come lacerto che permette la sopravvivenza di un testo, o, più specificamente, di un personaggio, viene dunque superata, di fronte alle molteplici possibilità che un'opera di citazioni offre alla lettura. Il Libro dei Libri (si legge ancora) è infatti la testimonianza «di una sola mitopea gigantesca, scritta da una sola innumerevole mano», un luogo nel quale i personaggi «fra loro si amassero, colluttassero, gridando chiedessero a tutti di vivere e somigliarci $»^{38}$. È interessante sottolineare che Bufalino parla, in questa occasione, di «anagrafe» dei personaggi: espressione identica a quella introdotta nel racconto La visione di Basilio ovvero La battaglia dei tarli e degli eroi ${ }^{39}$, nel quale è narrato il tentativo di porre a salvezza una «centuria» di libri, minacciati da invincibili tarli.

Proposta nelle righe dell'incipit dell'introduzione del Dizionario, l'idea della salvezza del sapere scivola in secondo piano, nella parte finale, per lasciare emergere un'osservazione che è ancora una volta vicina (e ancora una volta occorre usare l'avverbio paradossalmente) a quella di Benjamin, pur nella distanza tra le esperienze personali, le letture formative, le intenzioni di scrittura dei due scrittori: il libro di citazioni come libro del presente per il lettore del presente. 
il Dizionario dei personaggi di romanzo, Bufalino offre infatti al lettore un'ulteriore esperienza, oltre quella più consueta di trovar piacere nel riconoscimento di un testo già incontrato, o quella del riconoscimento di una citazione nascosta nel commento preliminare. Le voci del catalogo (Inventario di tipi romanzeschi, secondo un'ennesima definizione, collocata come titolo di uno dei vari scritti posti da Bufalino come apparati introduttivi al Dizionario ${ }^{40}$ ), sebbene siano testi 'chiusi' se considerati in rapporto all'opera dalla quale provengono, possono tuttavia essere riutilizzate da ciascun lettore per personali «divertenti bricolages culturali» ${ }^{41}$. I profili dei personaggi, offerti come materiali narrativi, permettono la costruzione di un personale mosaico narrativo, cioè la composizione scomposizione di nuove trame in una personale narrazione nella quale la combinazione di figure diverse, di tipi diversi, di costumi diversi, può dare vita a un testo nuovo. Le nuove narrazioni costruite mentalmente dal lettore - che possono avere titoli simili a quelli delle citazioni false dell'ingegnere di Babele - nascono dagli incroci di personaggi diversi, che, già noti per altre storie, sono pronti a entrare in storie nuove: Pinocchio che incontra Moll Flanders, per esempio, attraversando entrambi esperienze socialmente difficili per arrivare a una riconosciuta «normalità».

Lo stesso Bufalino, nei suoi brevi commenti introduttivi, suggerisce relazioni e legami tra personaggi e figure del mondo reale, spaziando dalla letteratura all'arte al cinema, indicando rimandi, collegamenti, fili che uniscono le esperienze di lettura e di spettacolo del lettore, a questo punto sollecitato ad essere lui stesso l'animatore dei collegamenti proposti o a instaurarne altri.

Alcuni pochi esempi lo possono confermare. «Renato» di Chateaubriand è un «seducente malato di inesistibili e tuttavia mortali affezioni», e la sua presentazione introduce subito un sottile richiamo che fa incontrare a distanza altre figure letterarie: "Quel che ci vuole per sedurre Marcel ragazzo e far sorridere Madame de Villaparisis...» ${ }^{42}$. La «mediocre mitologia» di Margherita Gautier - «I balli, le carrozze agli Champs Élysées [...], le vincite favolose al giuoco (trecento luigi in mezz'ora!), le mondane ingioiellate, i ventagli, i colpi di tosse nei fazzoletti di batista, i padri che dicono ai figli "Signore"...» - assume un particolare «profumo», «Specialmente se Margherita si fa prestare gli occhi grandi di Greta Garbo, o mormora "Dite alla giovane" con la voce di Maria Callas»" ${ }^{43}$. La figura di Madame Arnoux dell'Educazione sentimentale - «tenera e grave», che «reca nei salotti di metà secolo la malinconia di una bellezza minacciata dagli anni» - è «un Renoir firmato» ${ }^{44}$, mentre Federico Moreau è «irresoluto, quasi cecovianamente, fra slanci e prosa $»^{45}$. La confessione di Michel, nell'Immoralista di Gide, ricorda invece che «Un'angoscia che scrive bene non è mai veramente totale, così Valéry accusava addirittura Pascal», e rivela che il protagonista si è nutrito «à la carte in un locale alla moda, chez Nietzsche o chez Wilde» ${ }^{46}$. O, ancora, «I personaggi dell'Ulysses, parola di Svevo, camminano "col teschio scoperchiato": non così Zeno Cosini», che «quanto più sembra frugarsi e svelarsi, tanto più si nasconde dietro preziose malefedi $\mathrm{e}$ schermi d'umore ${ }^{47}$.

31 Nelle ultime righe dell'«Avvertenza» Bufalino, parla dei suoi brevi commenti introduttivi (che chiama «schede») come di «lapidi miliari [...] di un pellegrinaggio autour de sa chambre, dove la stanza e la Mecca sono, naturalmente, la biblioteca». Il Libro dei Libri, il Dizionario delle citazioni sono il catalogo di una biblioteca che, cadute le ragioni della storia, ciascun lettore è invitato a considerare come chambre à louer: una camera, tuttavia, da personalizzare per potersi muovere, in essa, come autour de sa chambre, come dentro una propria biblioteca. 
Dalla riflessione sulla storia e sulla sua conservazione dei testi del passato si è arrivati alla fine alla riflessione di Bufalino sulla letteratura. La proposta finale di lettura del Dizionario si pone come una delle numerose riflessioni di teoria della letteratura che, in particolare nella seconda metà del Novecento, sottolineano che ogni testo è il risultato di una riscrittura di materiali preesistenti e ricorrenti nelle pagine letterarie di ogni tempo. Nell'«Avvertenza» al Dizionario Bufalino ricorda l'affermazione di Musil «secondo la quale gli annali letterari non sarebbero che una ininterrotta catena di plagi attraverso i secoli ${ }^{48}$ : ma lo stesso Bufalino potrebbe modificarla con "un'ininterrotta catena di citazioni".

\section{NOTE}

1. Gesualdo Bufalino, L'ingegnere di Babele, in Id., L'uomo invaso e altre invenzioni, Milano, Bompiani, 1986, pp. 65-71. Da questa prima edizione le citazioni saranno indicate in nota con la sigla IB. La raccolta è stata poi ripubblicata in G. Bufalino, Opere 1981.1988, introduzione di M. Corti, a cura di M. Corti e F. Caputo, Milano, Bompiani, 1992. Accostando L'ingegnere di Babele al racconto Le visioni di Basilio ovvero la battaglia dei tarli e degli eroi, che lo segue immediatamente nella raccolta L'uomo invaso, Giuseppe Traina ha parlato di «dittico visionario e borgesiano contrassegnato dal tema della bibliofilia» (G. Traina, «Introduzione», in G. Bufalino, L'uomo invaso e altre invenzioni, introduzione di G. Traina, disegni di V. Vitali, Comiso, Fondazione Gesualdo Bufalino, 2016, p. 18).

2. IB, p. 65.

3. H. Arendt, Walter Benjamin: L'omino gobbo e il pescatore di perle, in Ead., Il futuro alle spalle, a cura di L. Ritter Santini, Il Mulino, Bologna 1981, p. 164.

4. Th. W. Adorno, Profilo di Walter Benjamin, in Id., Prismi. Saggi sulla critica della cultura, trad. it. di C. Mainoldi, Einaudi, Torino 1972, in particolare a p. 245.

5. Pietro Citati aveva intitolato un suo articolo Bufalino cannibale, divoratore di libri ("Corriere della Sera», 22 aprile 1988, p. 3).

6. Nel saggio Citazione come oblio, Gabriele Scaramuzza mostra con precisione come in Benjamin la citazione non abbia un valore ermeneutico, tanto è vero che le parole citate vengono inserite senza alcun controllo filologico. Si veda G. Scaramuzza, Citazione come oblio, in «Leitmotiv», $\mathrm{n}^{\circ} 1$, 2002, pp. 11-23, all'indirizzo: <www.ledonline.it/leitmotiv/>. In queste pagine anche ulteriori e significativi riferimenti bibliografici.

7. Si veda la sua edizione italiana in W. Benjamin, Franz Kafka. Per il decimo anniversario della sua morte, in Id., Angelus Novus. Saggi e frammenti, traduzione e introduzione di R. Solmi, Torino, Einaudi, 1962 (poi più volte riedita).

8. G. Scaramuzza, Citazione come oblio, cit., p. 17.

9. Ivi, p. 22.

10. Ivi, p. 21.

11. IB, p. 68.

12. Ibid.

13. IB, p. 67. Alcune brevi osservazioni su questo punto si leggono in A. Caputo, Arte della variazione. I racconti di Gesualdo Bufalino, in «Parole Rubate», fascicolo $\mathrm{n}^{\circ}$ 14, dicembre 2016, pp. 177-188 (<www.parolerubate.unipr.it/indici_php/fascicolo_14.php>).

14. IB, p. 68. 

15. IB, p. 69.
16. IB, p. 66.
17. IB, p. 67.
18. IB, p. 69.
19. IB, p. 70.
20. Ibid.

21. Maria Corti, «Introduzione» a G. Bufalino, Opere 1981.1988, cit., p. XVII.

22. Ivi, p. XVI.

23. Lo si vede bene nelle carte autografe del Guerin Meschino presentate in Maria Rita Mastropaolo, Vizi e vezzi del puparo: citazioni e criptocitazioni, in Oblio, $\mathrm{n}^{\circ} 21$, giugno 2016 (<www.progettoblio.com/downloads/Oblio,VI,21.pdf >).

24. Per altro Bufalino torna più volte sul tema delle citazioni, ma qui basti rimandare ai saggi più specifici raccolti in questo fascicolo di «Cahiers d'études italiennes» e alla loro bibliografia.

25. M. Corti, «Introduzione» a G. Bufalino, Opere 1981.1988, cit., p. XVI.

26. G. Traina, «Introduzione», cit., p. 19.

27. G. Bufalino, «Introduzione» a Id., Dizionario dei personaggi di romanzo. Da don Chisciotte all'Innominabile, Milano, il Saggiatore, 1982, dal quale si cita indicandolo con la sigla Dz nelle note bibliografiche. Il Dizionario è poi stato riproposto in edizione tascabile, con introduzione di Alberto Cadioli, Milano, Bompiani, 2000.

28. «Introduzione. Passione del personaggio», in Dz, p. 1.

29. Ibid.

30. Ivi, p. 2.

31. «Avvertenza», in Dz, p. 20.

32. «Don Chisciotte», in Dz, p. 35.

33. «Introduzione. Passione del personaggio», in Dz, p. 2.

34. Ibid.

35. Ibid.

36. Ivi, p. 14.

37. Ivi, p. 15.

38. Ibid.

39. Gesualdo Bufalino, La visione di Basilio ovvero La battaglia dei tarli e degli eroi, in Id., L'uomo invaso, cit., p. 77.

40. Dz, p. 21

41. «Avvertenza», in Dz, p. 20.

42. «Renato», in Dz, p. 113.

43. «Margherita Gautier», in Dz, p. 199.

44. «Federico Moreau e Madame Arnoux», in Dz, p. 260.

45. Ibid.

46. «Michel», in Dz, p. 344.

47. «Zeno Cosini», in Dz, p. 419.

48. «Avvertenza», in Dz, p. 20. 


\section{RIASSUNTI}

Il saggio esamina l'idea di Gesualdo Bufalino di un libro tutto composto di citazioni, di un Libro dei Libri, attraverso un confronto con Walter Benjamin e lo studio del Dizionario dei personaggi di romanzo.

This essay examines Bufalino's idea of a Book of Books, a book entirely made of quotations, through a comparison with Walter Benjamin's different and similar project, and an analysis of Bufalino's Dizionario dei personaggi di romanzo.

Cet essai examine l'idée émise par Bufalino d'un Livre des Livres, d'un livre entièrement composé de citations, à travers une comparaison avec le projet semblable et différent de Walter Benjamin, accompagnée d'une nouvelle lecture du Dizionario dei personaggi di romanzo.

\section{INDICE}

Parole chiave : citazione, lettura, riscrittura, Gesualdo Bufalino, Walter Benjamin Keywords : quotation, reading, rewriting, Gesualdo Bufalino, Walter Benjamin

Mots-clés : citation, lecture, réécriture, Gesualdo Bufalino, Walter Benjamin

\section{AUTORE}

\section{ALBERTO CADIOLI}

Università di Milano 